\title{
Trace-Level Screening of Chemicals Related to Clandestine Desomorphine Production with Ambient Sampling, Portable Mass Spectrometry
}

\author{
Seth E. Hall, Adam E. O'Leary, Zachary E. Lawton, Alessandra M. Bruno, \\ and Christopher C. Mulligan \\ Department of Chemistry, Illinois State University, Normal, IL, USA \\ Correspondence should be addressed to Christopher C. Mulligan; mulligan@ilstu.edu
}

Received 2 February 2017; Revised 10 June 2017; Accepted 28 June 2017; Published 1 August 2017

Academic Editor: Artur M. S. Silva

Copyright ( $\odot 2017$ Seth E. Hall et al. This is an open access article distributed under the Creative Commons Attribution License, which permits unrestricted use, distribution, and reproduction in any medium, provided the original work is properly cited.

Desomorphine is a semisynthetic opioid that is responsible for the psychoactive effects of a dangerous homemade injectable mixture that goes by street name "Krokodil." Desorption electrospray ionization (DESI) and paper spray ionization (PSI) are implemented on a portable mass spectrometer for the direct analysis of desomorphine and precursor reagent codeine from multiple substrates of potential relevance to clandestine drug laboratory synthesis and paraphernalia seizure. Minimal sample preparation required for analysis and portability of the instrument suggest the potential for rapid, on-site analysis of evidence, a highly desired benefit for forensic science and law enforcement practitioners. Both DESI-MS and PSI-MS can generate spectra consistent with preceding data obtained using traditional ionization methods, while demonstrating detection limits in the low- to sub-ng levels.

\section{Introduction}

The number of new designer drugs and older ones reappearing on the drug market is increasing and becoming a major public health concern. These designer substances are being used to substitute or mimic the effects of other controlled substances, while circumventing current drug policy and legality. Desomorphine $\left(\mathrm{C}_{17} \mathrm{H}_{21} \mathrm{NO}_{2}\right.$, dihydrodesoxymorphine) is the case of a reappearance of an older designer drug as an economical substitute for heroin, first observed in the Russian Federation and Ukraine in 2003 [1]. Desomorphine is a semisynthetic opioid that is responsible for the psychoactive effects of a dangerous homemade injectable mixture that goes by street name "Krokodil." This substance earned its street name "Krokodil" (crocodile in Russian) from $\alpha$-chlorocodide, a codeine derivative used in clandestine synthesis, and the physical discoloration and skin necrosis that resemble crocodile hide after prolonged usage [2]. The reemergence of desomorphine in the form of "Krokodil" is largely due to the history of heroin abuse in Russia, Ukraine, and other former Soviet countries that stems from their close proximity to Afghanistan, a major producer of opium $[2,3]$. As the availability of Afghanproduced heroin decreased, there was an increase in home production of injectable opioids to compensate for the lack of easily accessible heroin [4].

"Krokodil" poses a serious threat to users due to the severe symptoms and side effects but is also a concern with regard to its potential spread to other countries. It is believed that the use of "Krokodil" has already migrated to nearby countries including Poland, Czech Republic, France, Belgium, Sweden, Norway, and other European countries due to Russian immigration [5]. The process of "Krokodil" synthesis is nearly identical to the clandestine synthesis of methamphetamine from ephedrine [6,7], consisting of a simple extraction and reduction to obtain the opioid derivative. "Krokodil" is obtained using codeine as a starting material, which is commonly prescribed and sold in the pharmaceutical market in the form of tablets and syrups, and it can be synthesized using minimal equipment and synthetic steps [8]. The required reduction process is known as the Nagai synthesis route [7]. Given the simplicity of production and abundance of codeine precursor available, there is a growing concern for tracking the spread and usage trends of 
this drug. Drug monitoring agencies like the European Monitoring Centre for Drugs and Drug Addiction (EMCDDA) rely on analytical methods capable of screening and identifying forensic evidence relevant to "Krokodil" synthesis and distribution [9]. Current analytical methods typically involve the use of gas chromatography/mass spectrometry (GC/MS) or liquid chromatography/mass spectrometry (LC/MS) [10-13]. While being well-established analytical techniques, both are known to require extensive sample preparation and lengthy separation/analysis times and are primarily restricted to laboratory-based applications. The low throughput of these techniques combined with the inherent need to transport samples of interest to off-site facilities for analysis has produced significant backlogs of forensic evidence and delays in legal proceedings.

Ambient ionization mass spectrometric techniques such as desorption electrospray ionization mass spectrometry (DESI-MS) [14] and paper spray ionization mass spectrometry (PSI-MS) [15] have been shown to allow rapid screening of forensically relevant samples, while permitting trace-level detection with little to no sample preparation, as recently reviewed [16, 17]. Analysis via DESI-MS is achieved by directing a pneumatically assisted spray of charged solvent droplets towards a sample surface, promoting the extraction of analytes as secondary ions which are then sampled by atmospheric inlet MS systems [18-20]. PSI-MS combines the mechanism of ESI-MS and paper chromatography to achieve analysis by prompting spray ionization from a triangular paper substrate through saturation with a small aliquot $(<10 \mu \mathrm{L})$ of solution in combination with an applied voltage [15]. While DESI and PSI are regarded for their rapidity and decreased sample preparation, coupling these techniques with portable MS systems has the capability to provide massive gains in throughput by allowing evidence screening to occur on-site. Further, recent work involving portable MS instrumentation showed that ambient ionization methods could be used to successfully monitor clandestine methamphetamine production using the Nagai route similar to "Krokodil" [21], as well as being used to screen for relevant residues for common paraphernalia types [22].

Herein, we employ DESI and PSI in combination with a portable MS system for the analysis of desomorphine and its precursor codeine to show applicability to this important emerging drug. Screening of trace residues of desomorphine and codeine was conducted from surfaces commonly used or found in the storage, transport, and clandestine synthesis, reporting representative limits of detection. This work serves to show the utility of such an application to provide data of probative evidentiary value from a variety of forensic scenarios, ranging from reaction precursors, clandestine laboratory installations, seized bulk drugs, and trace evidence.

\section{Materials and Methods}

2.1. Sample Preparation. Solutions of desomorphine and codeine were obtained as $1.0 \mathrm{mg} / \mathrm{mL}$ analytical standards from Cerilliant Corp. (Round Rock, TX, USA) and serially diluted in methanol to desired concentrations of $0.10,0.010$, 0.0010 , and $0.00010 \mathrm{mg} / \mathrm{mL}$. Solutions were then spotted in predetermined amounts via adjustable micropipette onto porous Teflon well slides (Prosolia Inc., Indianapolis, IN, USA) for DESI analysis or Whatman chromatography paper (Fisher Scientific, Inc., Hampton, NH, USA) for PSI analysis according to the relationship that $1 \mu \mathrm{L}$ of deposited standard solution contains a known mass of analyte in nanograms proportional to the concentration prepared. For example, $150 \mathrm{ng}$ of analyte was deposited by spotting a $1.5 \mu \mathrm{L}$ aliquot of a $0.10 \mathrm{mg} / \mathrm{mL}$ solution. These aliquots were then dried for $\sim 5 \mathrm{~min}$ and analyzed directly with the chosen ionization method.

For limits of detection studies, known masses of desomorphine or codeine were deposited onto surfaces of interest by spotting $1 \mu \mathrm{L}$ aliquots of each solution, followed by drying to remove bulk solvent in order to simulate surface-bound residues. Surfaces relevant to clandestine synthesis and storage included glass, steel, enameled cookware, polyethylene terephthalate (PET) prescription bottles, and Teflon-coated cookware. In addition, a Tylenol 3 pill (e.g., acetaminophen and codeine) was also investigated to simulate a potential source of codeine precursor for the synthesis of desomorphine. Prior to analysis, the Tylenol 3 pill was slightly pulverized using a razor blade followed by immediate analysis with DESI and/or PSI.

Sampling of desomorphine and codeine residues from clandestine relevant surfaces with DESI-MS was performed by swabbing a $\sim 9 \mathrm{~cm}^{2}$ area of the surface of interest near the location of analyte deposition. Swabs utilized consisted of polyurethane foam (Berkshire Corp., Great Barrington, MA, USA) moistened with a small amount spray solvent, $\mathrm{H}_{2} \mathrm{O} / \mathrm{MeOH}(1: 1, \mathrm{v} / \mathrm{v})$ with $0.1 \%(\mathrm{v} / \mathrm{v})$ formic acid, to assist in the collection of surface residues. Swabs were immediately introduced into the DESI source after collection.

For analysis with PSI-MS, Whatman chromatography paper cut into $10 \mathrm{~mm} \times 5 \mathrm{~mm}$ isosceles triangles was employed as both the swabs and spray substrate. Prior to analysis, each triangle was saturated with methanol, similar to the protocol employed for DESI analysis, and used to swab the surface of interest. After swabbing, the paper triangle was attached to high voltage clamping electrode with the triangular apex pointing axially towards the inlet capillary of the MS system. A $4 \mathrm{kV}$ voltage was then supplied to the electrode, followed by the addition of $2 \mu \mathrm{L}$ of spray solvent, $\mathrm{H}_{2} \mathrm{O} / \mathrm{MeOH}$ $(1: 1, \mathrm{v} / \mathrm{v})$ with $0.1 \%(\mathrm{v} / \mathrm{v})$ formic acid, to generate analyte ions.

2.2. Portable Mass Spectrometer and Experimental Variables. All experiments were performed using a FLIR Systems AI-MS 1.2 cylindrical ion trap (CIT) mass spectrometer (FLIR Mass Spectrometry, West Lafayette, IN, USA), as previously reported when used in forensic applications [2325]. The instrument possesses a factory-produced DESI source with solvent delivery provided by an on-board syringe pump. Cartridge-based helium damping gas for collisionally induced dissociation during tandem MS experiments is incorporated in the overall instrument design and delivered at 40 psi, whereas $\mathrm{N}_{2}$ nebulizing gas was provided by a standalone gas canister or a small SCBA tank for field usage at a constant pressure of 100 psi. Note that only DESI utilizes nebulizing gas in its ionization process. Each experiment was 


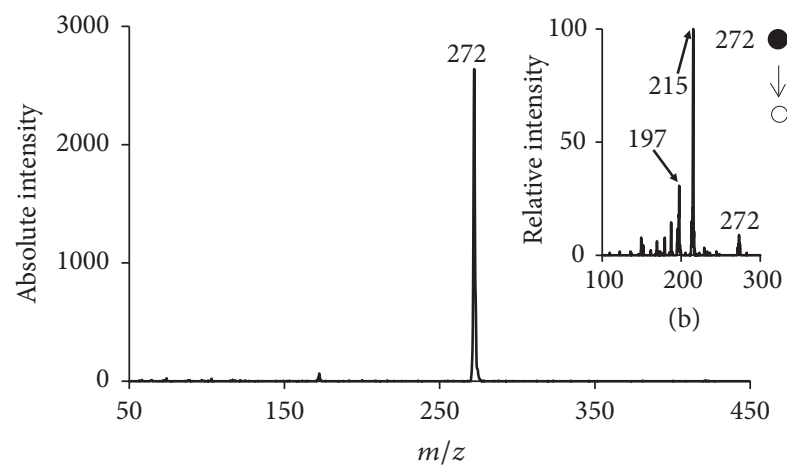

(a)

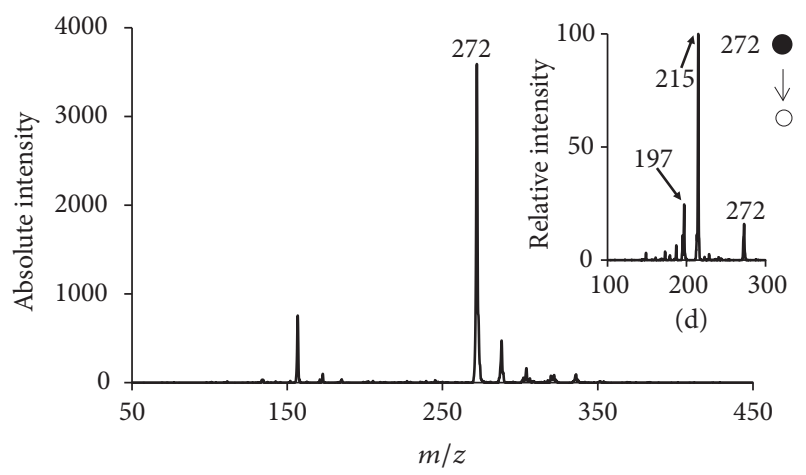

(c)

FIgURE 1: Ambient MS analyses of desomorphine on the FLIR Systems AI-MS 1.2. (a) DESI-MS spectrum. (b) DESI-MS/MS of protonated desomorphine. (c) PSI-MS spectrum. (d) PSI-MS/MS of protonated desomorphine.

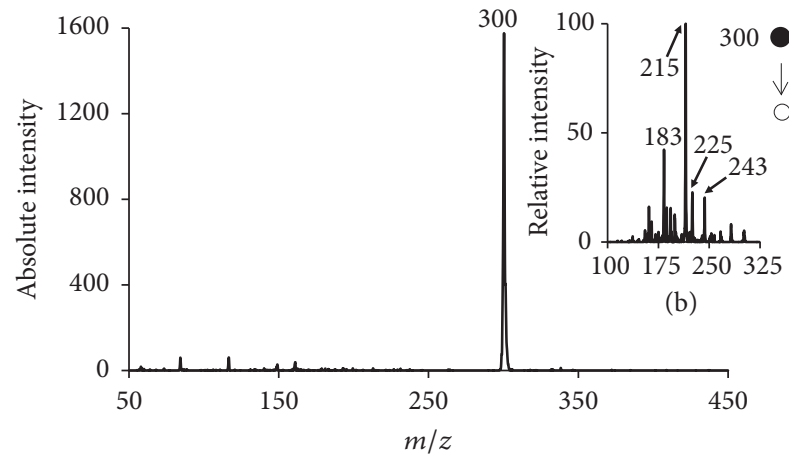

(a)

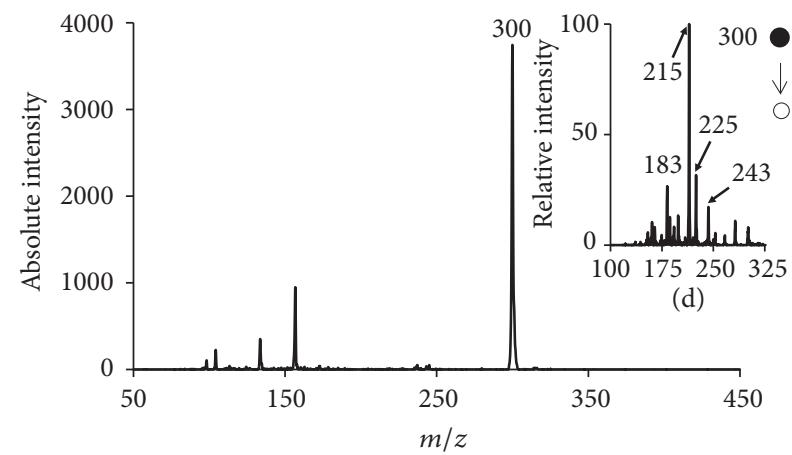

(c)

FIgURE 2: Ambient MS analyses of codeine on the FLIR Systems AI-MS 1.2. (a) DESI-MS spectrum. (b) DESI-MS/MS of protonated codeine. (c) PSI-MS spectrum. (d) PSI-MS/MS of protonated codeine.

performed using both DESI and PSI ionization. All DESI studies utilized a spray solvent composition of $\mathrm{H}_{2} \mathrm{O} / \mathrm{MeOH}$ $(1: 1, \mathrm{v} / \mathrm{v})$ with $0.1 \%(\mathrm{v} / \mathrm{v})$ formic acid and a solvent flow rate of $3 \mu \mathrm{L} / \mathrm{min}$. All PSI studies utilized a $2 \mu \mathrm{L}$ aliquot of the previously mentioned spray solvent composition for analysis. A $4 \mathrm{kV}$ source voltage was used for both DESI and PSI, supplied by the on-board voltage sources of the AI-MS 1.2.

All analytical scenarios presented herein were examined with both DESI-MS and PSI-MS in positive-ion mode. Pertinent operating conditions for ion collection and focusing via the atmospheric pressure capillary inlet include capillary voltage of $60 \mathrm{~V}$, tube lens voltage of $150 \mathrm{~V}$, and skimmer voltage of $17 \mathrm{~V}$. The inlet capillary of the AI-MS 1.2 is not temperature controlled. Ions were collected via ion gating for $100 \mathrm{~ms} / \mathrm{scan}$, and 10 scans/average were utilized for collected spectra.

\section{Results and Discussion}

3.1. MS and $M S^{2}$ of Target Analytes and Limits of Detection (LODs). In order to demonstrate detection capability with the proposed methods, representative positive-ion DESI and PSI-MS data acquired with MS and $\mathrm{MS}^{2}$ scan modes were collected for the target analytes. DESI and PSI-MS data obtained for desomorphine is shown in Figure 1. It can be seen that both ionization methods result in a single parent ion [desomorphine $+\mathrm{H}]^{+}$at $m / z 272$ (Figures 1(a) and $1(\mathrm{c}))$, as well as characteristic fragments at $m / z 215$ and $m / z 197$, corresponding to proposed losses of $\mathrm{C}_{3} \mathrm{H}_{7} \mathrm{~N}$ and $\mathrm{C}_{3} \mathrm{H}_{7} \mathrm{NH}_{2} \mathrm{O}$ (as seen in Figures 1(b) and 1(d)). Similar fragmentation pathways have been recently reported using high-resolution LC-MS/MS [12], and high-resolution MS (HR-MS) studies performed in house returned high mass accuracy $(\leq 2.66 \mathrm{ppm})$ for the proposed assignments; HRMS data obtained and associated error can be seen in the Supplementary Material (in Supplementary Material available online at https://doi.org/10.1155/2017/8571928). Representative DESI-MS and PSI-MS data for the desomorphine precursor, codeine, can be seen in Figure 2. Both ionization sources yield a single parent ion, [codeine $+\mathrm{H}]^{+}$at $\mathrm{m} / z$ 300 (Figures 2(a) and 2(c)), with characteristic fragments at $m / z 183,215,225$, and 243 corresponding to proposed losses of $\mathrm{C}_{13} \mathrm{H}_{11} \mathrm{O}, \mathrm{C}_{4} \mathrm{H}_{7} \mathrm{NO}, \mathrm{C}_{3} \mathrm{H}_{9} \mathrm{NO}$, and $\mathrm{C}_{3} \mathrm{H}_{7} \mathrm{~N}$, respectively (as seen in Figures 2(b) and 2(d)); these $\mathrm{MS}^{2}$ transitions and associated losses are similar to those reported in the 
TABLE 1: Limits of detection (LODs) obtained for the target surfaces studied.

\begin{tabular}{lcccc}
\hline Surface & \multicolumn{2}{c}{ DESI-MS } & \multicolumn{2}{c}{ PSI-MS } \\
& Desomorphine & Codeine & Desomorphine & Codeine \\
\hline Printed Teflon slide & $0.50 \mathrm{ng}$ & $0.90 \mathrm{ng}$ & - & \\
Steel & $90 \mathrm{ng}$ & $100 \mathrm{ng}$ & $4.0 \mathrm{ng}$ & $8.0 \mathrm{ng}$ \\
Nonstick cookware & $100 \mathrm{ng}$ & $150 \mathrm{ng}$ & $2.0 \mathrm{ng}$ & $10 \mathrm{ng}$ \\
Enamel cookware & $200 \mathrm{ng}$ & $200 \mathrm{ng}$ & $2.5 \mathrm{ng}$ & $4.0 \mathrm{ng}$ \\
PET bottle & $100 \mathrm{ng}$ & $270 \mathrm{ng}$ & $2.0 \mathrm{ng}$ & $3.5 \mathrm{ng}$ \\
Glass & $150 \mathrm{ng}$ & $350 \mathrm{ng}$ & & $3.0 \mathrm{ng}$ \\
\hline
\end{tabular}

literature [26]. Of note, comparison of the MS spectral intensities obtained for the target analytes using both ionization methods demonstrates higher signal intensity with PSI for both analytes.

Given the direct analysis capabilities of both DESI-MS and PSI-MS, limits of detection (LODs) were determined for the analysis of surfaces commonly encountered in clandestine synthesis laboratories and as seized paraphernalia (i.e., steel and enamel cookware, PET bottles, and glass). For LOD studies, a $1 \mu \mathrm{L}$ aliquot of target analyte was applied to a surface of interest followed by swabbing with the appropriate transfer swab or substrate and immediate analysis in DESI or PSI. Results of the LOD studies are summarized in Table 1. Overall, LODs ranged in the low- to sub-ng magnitude demonstrating the capability of trace-level detection using both ionization methods. Direct analysis of target analytes from printed Teflon slides via DESI-MS displayed sub-ng LODs, while analysis of substrates resulted in LODs between 90 and $150 \mathrm{ng}$. Of note, analysis from the target surfaces with PSIMS yielded LODs approximately two orders of magnitude lower than DESI. It is likely that the higher LOD magnitudes experienced with direct analysis via DESI are a consequence of its two-step ionization mechanism [20], which likely contributes to its overall lower ion signal observed.

3.2. Analysis of Simulated Forensic Evidence. In the event of encountering authentic evidence relating to clandestine drug manufacture and distribution, it is quite likely that target analyte(s) will exist as mixtures in potentially complex matrices, such as inactive ingredients found in pharmaceutical precursors, various cutting agents observed in street-level drugs, and contaminants stemming from nonsterile clandestine glassware or storage containers. This is especially the case for the haphazard synthesis of desomorphine, in which there is a high possibility of encountering an incomplete reaction, specifically a slurry containing desomorphine along with unreacted codeine precursor. In order to demonstrate the robustness of the proposed method, multicomponent sample analysis of both substances was demonstrated for both DESI and PSI. Figure 3(a) displays a positive-ion DESI-MS spectrum of a mixture containing $10 \mu \mathrm{g}$ each of desomorphine $(\mathrm{m} / z$ 272) and codeine $(\mathrm{m} / z$ 300) swabbed from Tefloncoated cookware, in which intense ion signatures can be seen for each analyte. This is also supported in the positive-ion PSI-MS spectrum of the same mixture containing $900 \mathrm{ng}$ of

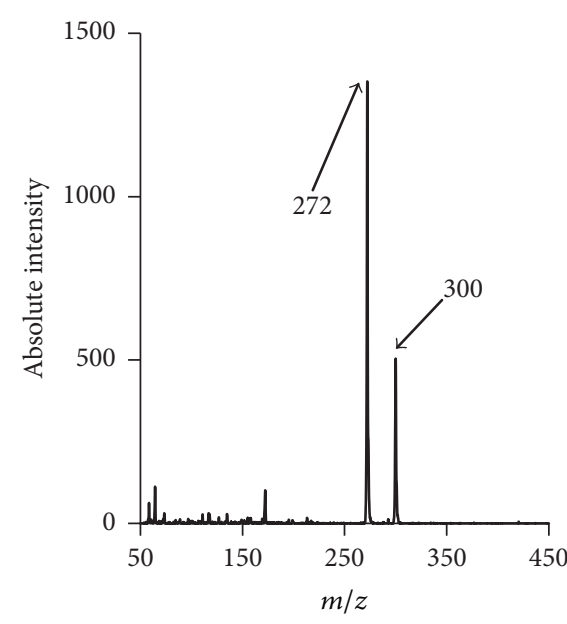

(a)

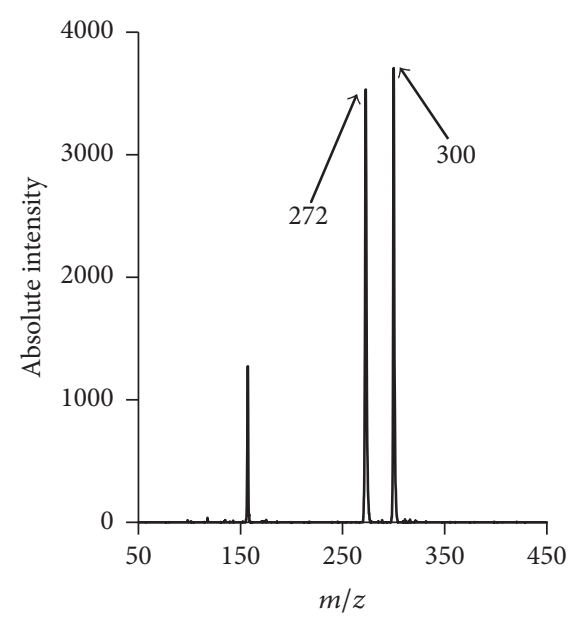

(b)

FIGURE 3: DESI and PSI-MS analysis of a simulated mixture sampled from Teflon-coated cookware. (a) DESI-MS spectrum of $10 \mu \mathrm{g}$ desomorphine and $10 \mu \mathrm{g}$ codeine. (b) PSI-MS spectrum of $900 \mathrm{ng}$ desomorphine and $600 \mathrm{ng}$ codeine.

desomorphine and $600 \mathrm{ng}$ of codeine (as seen in Figure 3(b)) with the only differing factor being a dramatic increase in spectral intensity due to the enhanced sensitivity of PSI. 


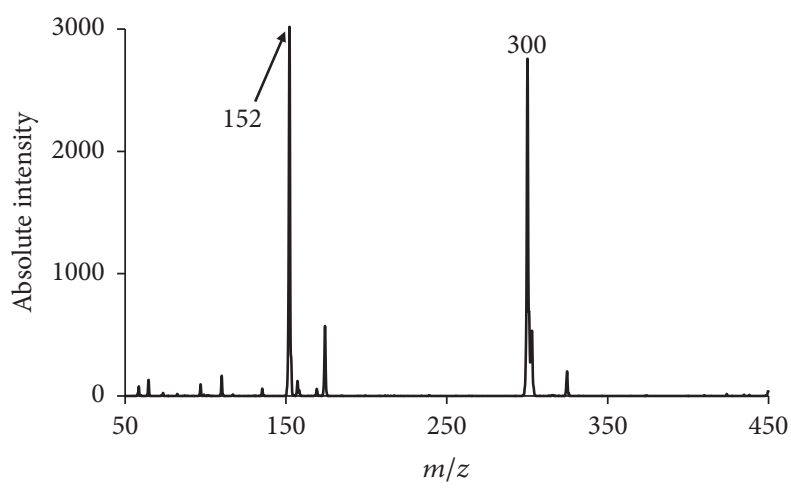

(a)

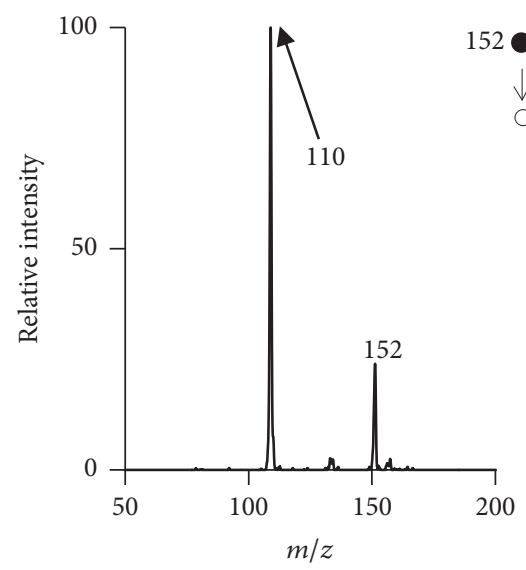

(c)

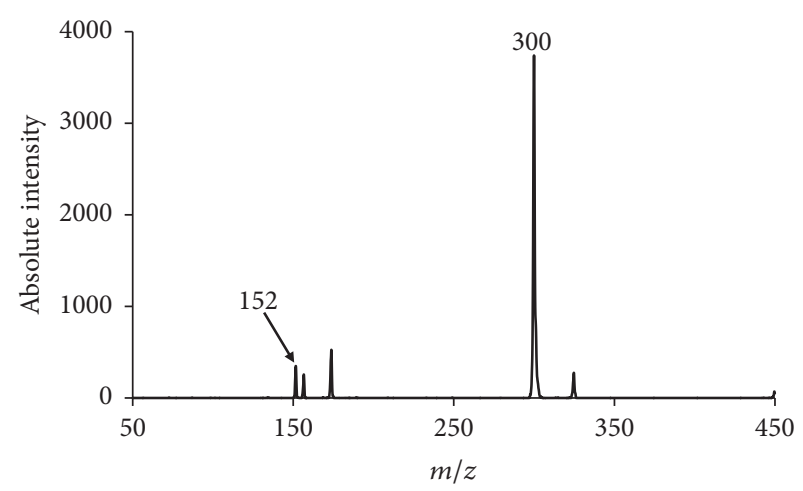

(b)

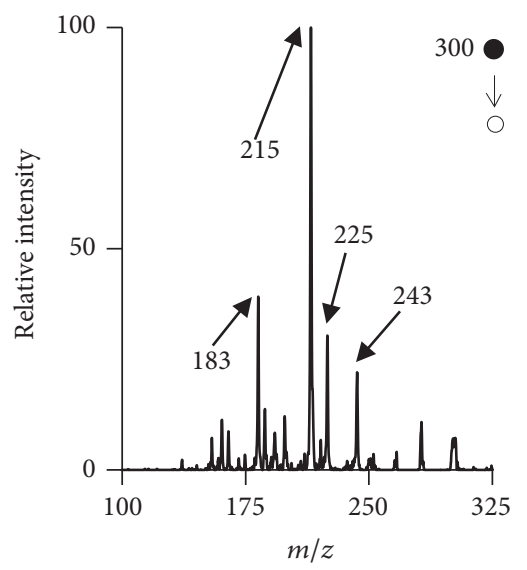

(d)

FIGURE 4: (a) DESI-MS spectrum of a Tylenol 3 tablet, giving rise to protonated acetaminophen and codeine. (b) PSI-MS spectrum of Tylenol 3. (c) MS/MS spectrum of protonated acetaminophen. (d) MS/MS spectrum of protonated codeine.

A highly desirable capability of on-site evidence screening methods would be to allow direct analysis of authentic forensic evidence with minimal sample preparation. In relation to the synthesis of desomorphine, the codeine precursor is needed as a starting reagent, typically obtained through the extraction of codeine-containing pharmaceutical tablets. To demonstrate the ability to directly screen authentic forensic samples of interest to "Krokodil" production with minimal preparation, DESI and PSI-MS were used to analyze a Tylenol 3 prescription pharmaceutical tablet; the active ingredients in Tylenol 3 are acetaminophen $(325 \mathrm{mg})$ and codeine $(30 \mathrm{mg})$. As seen in Figures 4(a) and 4(b), both ionization methods yield a single-ion signature for codeine at high spectral intensity, whereas the signal intensity for acetaminophen $(m / z 152)$ varied between spectra. $\mathrm{MS}^{2}$ analysis of the protonated parent ion for acetaminophen at $m / z 152$ yielded a single fragment transition at $m / z 110$, as seen in Figure 4(c), corresponding to a loss of $\mathrm{COCH}_{2}$, as reported in the literature [27]. Characteristic $\mathrm{MS}^{2}$ fragments were observed for codeine as well (Figure 4(d)), matching well that obtained in previous experiments involving analytical standards.

\section{Conclusions}

DESI and PSI-MS ionization sources were coupled with a portable ion trap mass spectrometer to demonstrate the direct analysis capabilities of desomorphine and its precursor codeine. The validity of this method was evaluated through the analysis of trace-level surface-bound residues, yielding low- to sub-ng detection limits from several substrates common to clandestine synthesis. Characteristic fragmentation similar to those reported in the literature and obtained on high-resolution MS instrumentation was achieved, providing confirmation of the target analytes. Due to the varying nature of forensic evidence with regard to chemical complexity, the applicability of the proposed method to multicomponent sample analysis was demonstrated, yielding high chemical specificity. Analysis of an authentic source of codeine precursor (in the form of prescription pharmaceutical tablets) was also demonstrated, supporting the proposed method's ability of directly analyzing forensic evidence with minimal sample preparation. 


\section{Disclosure}

The opinions, findings, and conclusions or recommendations expressed in this publication are those of the authors and do not necessarily reflect those of the Department of Justice.

\section{Conflicts of Interest}

The authors declare that there are no conflicts of interest regarding the publication of this paper.

\section{Acknowledgments}

This project was supported by Award nos. 2011-DN-BXK552 and 2015-IJ-CX-K011, awarded by the National Institute of Justice, Office of Justice Programs, U.S. Department of Justice. Molecular assignments for fragmentation spectra were made for select compounds with assistance from high resolution MS instrumentation acquired through support by the National Science Foundation MRI Program under Grant no. CHE 1337497.

\section{References}

[1] M. Gahr, R. W. Freudenmann, C. Hiemke, I. M. Gunst, B. J. Connemann, and C. Schönfeldt-Lecuona, "Desomorphine goes 'crocodile,' Journal of Addictive Diseases, vol. 31, no. 4, pp. 407412, 2012.

[2] J.-P. C. Grund, A. Latypov, and M. Harris, "Breaking worse: The emergence of krokodil and excessive injuries among people who inject drugs in Eurasia," International Journal of Drug Policy, vol. 24, no. 4, pp. 265-274, 2013.

[3] M. Gahr, R. W. Freudenmann, C. Hiemke, I. M. Gunst, B. J. Connemann, and C. Schönfeldt-Lecuona, "Krokodil': revival of an old drug with new problems," Substance Use and Misuse, vol. 47, no. 7, pp. 861-863, 2012.

[4] N. Abdala, J.-P. C. Grund, Y. Tolstov, A. P. Kozlov, and R. Heimer, "Can home-made injectable opiates contribute to the HIV epidemic among injection drug users in the countries of the former Soviet Union?" Addiction, vol. 101, no. 5, pp. 731-737, 2006.

[5] M. C. Van Hout, "Kitchen chemistry: A scoping review of the diversionary use of pharmaceuticals for non-medicinal use and home production of drug solutions," Drug Testing and Analysis, vol. 6, no. 7-8, pp. 778-787, 2014.

[6] A. Allen and T. S. Cantrell, "Synthetic reductions in clandestine amphetamine and methamphetamine laboratories: A review," Forensic Science International, vol. 42, no. 3, pp. 183-199, 1989.

[7] V. Kunalan, W. J. Kerr, and N. Nic Daéid, "Investigation of the reaction impurities associated with methylamphetamine synthesized using the Nagai method," Analytical Chemistry, vol. 84, no. 13, pp. 5744-5752, 2012.

[8] E. A. Alves, J.-P. C. Grund, C. M. Afonso, A. D. P. Netto, F. Carvalho, and R. J. Dinis-Oliveira, "The harmful chemistry behind krokodil (desomorphine) synthesis and mechanisms of toxicity," Forensic Science International, vol. 249, pp. 207-213, 2015.

[9] D. V. Thekkemuriyi, S. G. John, and U. Pillai, “'Krokodil'-a designer drug from across the Atlantic, with serious consequences," American Journal of Medicine, vol. 127, no. 3, pp. e1-e2, 2014.
[10] S. A. Savchuk, S. S. Barsegyan, I. B. Barsegyan, and G. M. Kolesov, "Chromatographic study of expert and biological samples containing desomorphine," Journal of Analytical Chemistry, vol. 63, no. 4, pp. 361-370, 2008.

[11] E. A. Alves, J. X. Soares, C. M. Afonso et al., "The harmful chemistry behind 'krokodil': street-like synthesis and product analysis," Forensic Science International, vol. 257, pp. 76-82, 2015.

[12] L. H. J. Richter, Y. R. Kaminski, F. Noor, M. R. Meyer, and H. H. Maurer, "Metabolic fate of desomorphine elucidated using rat urine, pooled human liver preparations, and human hepatocyte cultures as well as its detectability using standard urine screening approaches," Analytical and Bioanalytical Chemistry, vol. 408, no. 23, pp. 6283-6294, 2016.

[13] D. H. Â. Florez, A. M. dos Santos Moreira, P. R. da Silva et al., "Desomorphine (Krokodil): an overview of its chemistry, pharmacology, metabolism, toxicology and analysis," Drug and Alcohol Dependence, vol. 173, pp. 59-68, 2017.

[14] R. G. Cooks, Z. Ouyang, Z. Takats, and J. M. Wiseman, "Ambient mass spectrometry," Science, vol. 311, no. 5767, pp. 15661570,2006

[15] J. Liu, H. Wang, N. E. Manicke, J.-M. Lin, R. G. Cooks, and Z. Ouyang, "Development, characterization, and application of paper spray ionization," Analytical Chemistry, vol. 82, no. 6, pp. 2463-2471, 2010.

[16] D. N. Correa, J. M. Santos, L. S. Eberlin, M. N. Eberlin, and S. F. Teunissen, "Forensic chemistry and ambient mass spectrometry: a perfect couple destined for a happy marriage?" Analytical Chemistry, vol. 88, no. 5, pp. 2515-2526, 2016.

[17] R. Javanshad and A. R. Venter, "Ambient ionization mass spectrometry: real-time, proximal sample processing and ionization," Analytical Methods, 2017.

[18] N. Talaty, C. C. Mulligan, D. R. Justes, A. U. Jackson, R. J. Noll, and R. G. Cooks, "Fabric analysis by ambient mass spectrometry for explosives and drugs," Analyst, vol. 133, no. 11, pp. 1532-1540, 2008.

[19] L. Luosujärvi, U.-M. Laakkonen, R. Kostiainen, T. Kotiaho, and T. J. Kauppila, "Analysis of street market confiscated drugs by desorption atmospheric pressure photoionization and desorption electrospray ionization coupled with mass spectrometry," Rapid Communications in Mass Spectrometry, vol. 23, no. 9, pp. 1401-1404, 2009.

[20] M. Morelato, A. Beavis, P. Kirkbride, and C. Roux, "Forensic applications of desorption electrospray ionisation mass spectrometry (DESI-MS)," Forensic Science International, vol. 226, no. 1-3, pp. 10-21, 2013.

[21] A. E. O'Leary, S. E. Hall, K. E. Vircks, and C. C. Mulligan, "Monitoring the clandestine synthesis of methamphetamine in real-time with ambient sampling, portable mass spectrometry," Analytical Methods, vol. 7, no. 17, pp. 7156-7163, 2015.

[22] A. M. Bruno, S. R. Cleary, A. E. O’Leary, M. C. Gizzi, and C. C. Mulligan, "Balancing the utility and legality of implementing portable mass spectrometers coupled with ambient ionization in routine law enforcement activities," Analytical Methods, 2017.

[23] K. E. Vircks and C. C. Mulligan, "Rapid screening of synthetic cathinones as trace residues and in authentic seizures using a portable mass spectrometer equipped with desorption electrospray ionization," Rapid Communications in Mass Spectrometry, vol. 26, no. 23, pp. 2665-2672, 2012. 
[24] A. E. O'Leary, H. Oberacher, S. E. Hall, and C. C. Mulligan, "Combining a portable, tandem mass spectrometer with automated library searching - an important step towards streamlined, on-site identification of forensic evidence," Analytical Methods, vol. 7, no. 8, pp. 3331-3339, 2015.

[25] Z. E. Lawton, A. Traub, W. L. Fatigante et al., "Analytical validation of a portable mass spectrometer featuring interchangeable, ambient ionization sources for high throughput forensic evidence screening," Journal of the American Society for Mass Spectrometry, vol. 28, no. 6, pp. 1048-1059, 2017.

[26] C. Poeaknapo, U. Fisinger, M. H. Zenk, and J. Schmidt, "Evaluation of the mass spectrometric fragmentation of codeine and morphine after ${ }^{13} \mathrm{C}$-isotope biosynthetic labeling," Phytochemistry, vol. 65, no. 10, pp. 1413-1420, 2004.

[27] T. Belal, T. Awad, and C. R. Clark, "Determination of paracetamol and tramadol hydrochloride in pharmaceutical mixture using HPLC and GC-MS," Journal of Chromatographic Science, vol. 47, no. 10, pp. 849-854, 2009. 

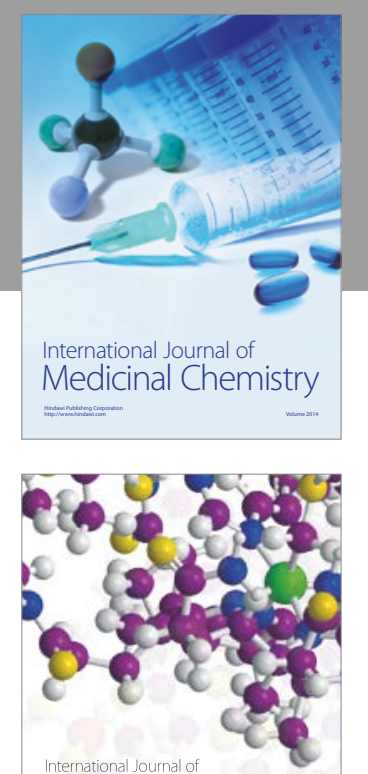

Carbohydrate Chemistry

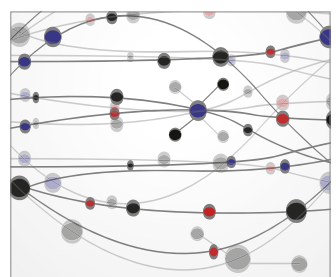

The Scientific World Journal
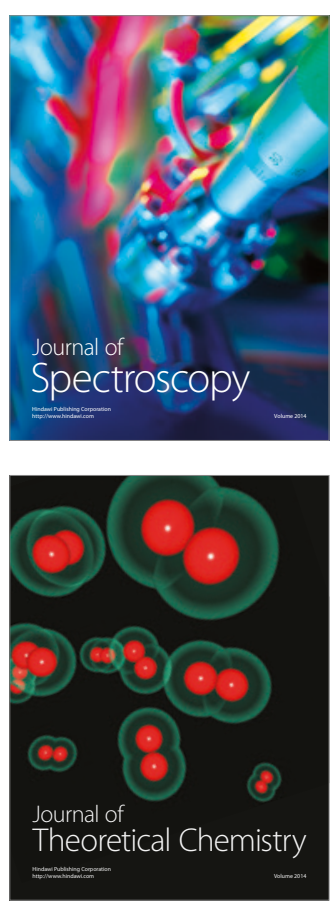
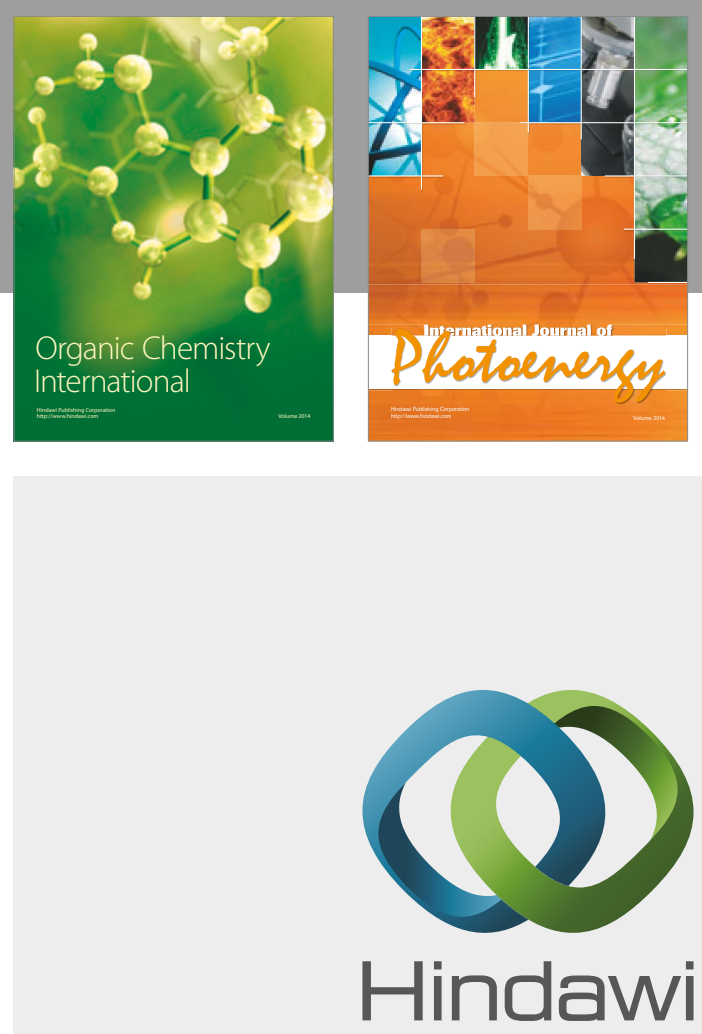

Submit your manuscripts at

https://www.hindawi.com

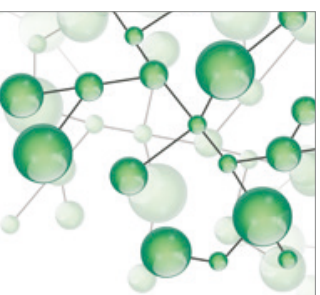

International Journal of

Inorganic Chemistry

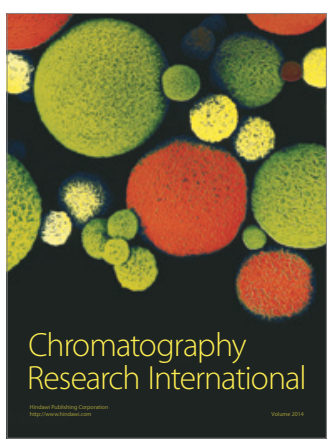

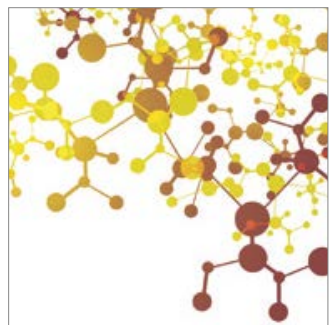

Applied Chemistry
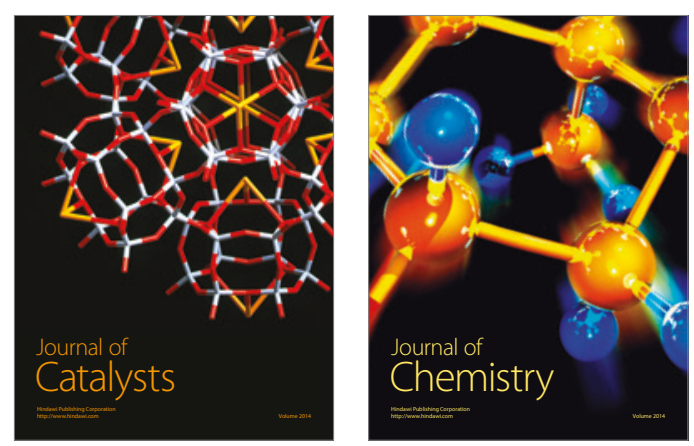
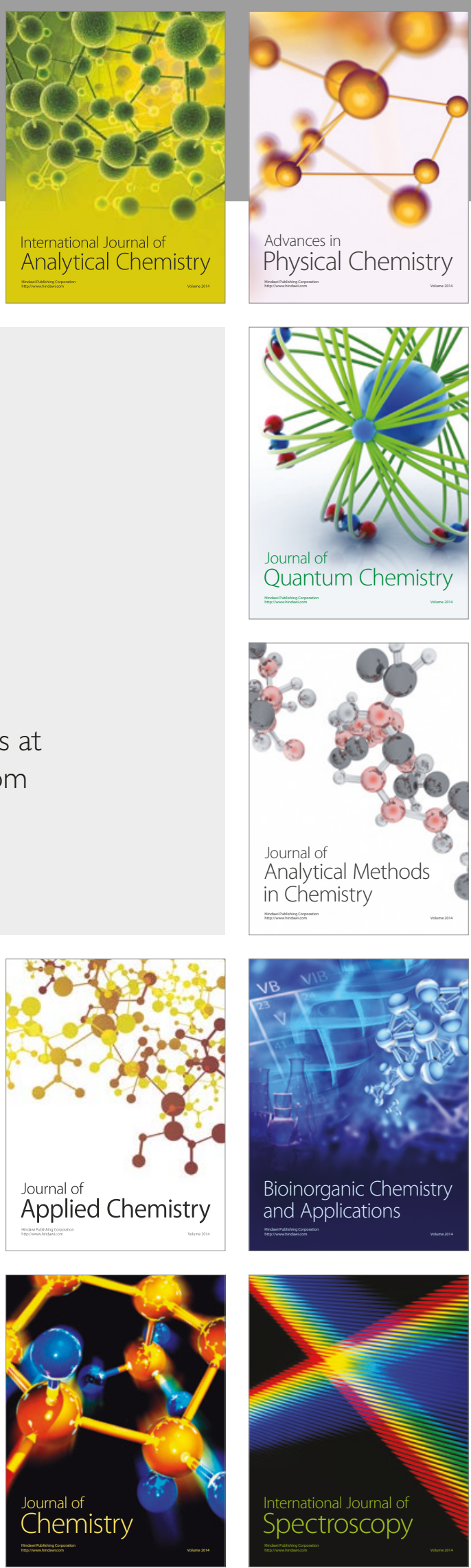